\title{
Proton and neutron skins and symmetry energy of mirror nuclei
}

\author{
M.K. Gaidarov ${ }^{\mathrm{a}, *}$, I. Moumene ${ }^{\mathrm{b}}$, A.N. Antonov ${ }^{\mathrm{a}}$, D.N. Kadrev ${ }^{\mathrm{a}}$, \\ P. Sarriguren ${ }^{\mathrm{c}}$, E. Moya de Guerra ${ }^{\mathrm{d}}$ \\ a Institute for Nuclear Research and Nuclear Energy, Bulgarian Academy of Sciences, Sofia 1784, Bulgaria \\ ${ }^{\mathrm{b}}$ High Energy Physics and Astrophysics Laboratory, Faculty of Science Semlalia, Cadi Ayyad University, P.O.B. 2390, \\ Marrakesh, Morocco \\ ${ }^{\mathrm{c}}$ Instituto de Estructura de la Materia, IEM-CSIC, Serrano 123, E-28006 Madrid, Spain \\ d Departamento de Estructura de la Materia, Física Térmica y Electrónica, and IPARCOS, Facultad de Ciencias \\ Físicas, Universidad Complutense de Madrid, Madrid E-28040, Spain
}

Received 24 September 2020; accepted 15 October 2020

Available online 20 October 2020

\begin{abstract}
The neutron skin of nuclei is an important fundamental property, but its accurate measurement faces many challenges. Inspired by charge symmetry of nuclear forces, the neutron skin of a neutron-rich nucleus is related to the difference between the charge radii of the corresponding mirror nuclei. We investigate this relation within the framework of the Hartree-Fock-Bogoliubov method with Skyrme interactions. Predictions for proton skins are also made for several mirror pairs in the middle mass range. For the first time the correlation between the thickness of the neutron skin and the characteristics related with the density dependence of the nuclear symmetry energy is investigated simultaneously for nuclei and their corresponding mirror partners. As an example, the Ni isotopic chain with mass number $A=48-60$ is considered. These quantities are calculated within the coherent density fluctuation model using Brueckner and Skyrme energy-density functionals for isospin asymmetric nuclear matter with two Skyrme-type effective interactions, SkM* and SLy4. Results are also presented for the symmetry energy as a function of $A$ for a family of mirror pairs from selected chains of nuclei with $Z=20, N=14$, and $N=50$. The evolution curves show a similar behavior crossing at the $N=Z$ nucleus in each chain and a smooth growing deviation when $N \neq Z$ starts. Comparison of our results for the radii and skins with those from the calculations based on high-precision chiral forces is made.
\end{abstract}

\footnotetext{
* Corresponding author.

E-mail address: gaidarov@inrne.bas.bg (M.K. Gaidarov).
} 
(C) 2020 Elsevier B.V. All rights reserved.

Keywords: Mirror nuclei; Nuclear structure models; Skyrme interactions; Nuclear skins; Energy density functionals; Symmetry energy

\section{Introduction}

The neutron-skin thickness represents a ground state property of finite nucleus that is a strong indicator of the isovector properties of effective nuclear interactions [1]. Its knowledge gives more insight into the properties of neutron-rich nuclei and neutron stars, and the equation of state (EOS) of asymmetric nuclear matter (ANM). The direct determination of the neutron-skin thickness usually involves the precise measurement of the root mean square (rms) radii of both charge and mass distributions. Electron-nucleus scattering has proven to be an excellent tool for the study of nuclear structure. In particular, it has accumulated much reliable information on the charge density distributions of stable nuclei. Therefore, it is believed that the new facilities in GSI [2,3] and RIKEN [4-6] will provide a good opportunity to study the charge density, and consequently the proton density distribution, of unstable nuclei by elastic electron scattering. In RIKEN-SCRIT the first elastic electron scattering experiment on the stable ${ }^{132} \mathrm{Xe}$ has already been performed [7]. Unfortunately, a measurement of the neutron density distributions to a precision and details comparable to that of the proton one is hardly possible. It turned out that to get information on the neutron-skin thickness one needs data obtained with probes having different sensitivities to the proton and neutron distributions.

The model-independent measurement of parity-violating asymmetry [8,9] (which is sensitive to the neutron distribution) in the elastic scattering of polarized electrons from ${ }^{208} \mathrm{~Pb}$ at JLAB within the PREX Collaboration [10,11] has provided the first electroweak observation of the neutron-skin thickness $0.33_{-0.18}^{+0.16} \mathrm{fm}$ in ${ }^{208} \mathrm{~Pb}$ (see also Ref. [12] for more discussion). A PREXII experiment has been approved [13] and it is expected to reach the $0.06 \mathrm{fm}$ sensitivity in the neutron radius of ${ }^{208} \mathrm{~Pb}$. Parity-violating experiments (CREX) are planned for the ${ }^{48} \mathrm{Ca}$ nucleus $[14,15]$.

Experimental difficulties in direct neutron-skin measurements and uncertainty about the sensitivity of mean-field models to isovector quantities [16] make alternative approaches desirable. Mahzoon et al. have proposed a method of determining the neutron rms radius and the neutronskin thickness of ${ }^{48} \mathrm{Ca}$ using a dispersive-optical-model (DOM) analysis of bound and scattering data to constrain the nucleon self-energy [17]. A best fit neutron skin of $0.249 \pm 0.023 \mathrm{fm}$ was deduced. Previously, applying the DOM the same authors considered the $N=Z$ system ${ }^{40} \mathrm{Ca}$ and extracted a very small, but negative, skin thickness of $-0.06 \mathrm{fm}$ [18]. It has been argued that while the proton and neutron distributions of ${ }^{40} \mathrm{Ca}$ are very similar and there is essentially no neutron skin, as expected, the magnitude of the sizable neutron skin of ${ }^{48} \mathrm{Ca}$ comes predominately from the $f_{7 / 2}$ orbital, reflecting its centrifugal barrier. The nonlocal DOM dispersive analysis of ${ }^{208} \mathrm{~Pb}$ has been also carried out, from which a neutron skin of $0.25 \pm 0.05 \mathrm{fm}$ was deduced [19]. A very recent systematic study of more nuclei has been performed in Ref. [20] using a newly-generalized version of the DOM. In addition, the neutron density distributions and the neutron-skin thickness in ${ }^{96} \mathrm{Zr}$ and ${ }^{96} \mathrm{Ru}$ nuclei have been probed with ultrarelativistic isobaric collisions [21] showing that they could be determined to a precision which may exceed those achieved by traditional low energy nuclear experiments. 
It has been shown in Ref. [22] that in the case of a perfect charge symmetry the neutron skin in a given nucleus can be obtained from the proton radii of mirror nuclei. Therefore, besides the planned JLAB experiment, measurements of mirror charge radii could be an alternative with a competitive precision. The necessary step after measuring the charge rms radii is to apply the relativistic and finite size corrections to deduce the point-proton rms radii. The correlations discussed in Ref. [22] between the neutron skin and the difference of the proton radii are determined for a particular mirror pair. This was realized by constructing 48 Skyrme functionals to predict different skins of ${ }^{208} \mathrm{~Pb}$ within a chosen range. Moreover, it was also shown that the difference in the charge radii of mirror nuclei is proportional to the slope of the symmetry energy $L$ at saturation density, even in the presence of the Coulomb corrections. The same findings have been confirmed in an approach based on a set of 14 relativistic energy density functionals (EDFs) spanning a wide region of values of $L$ [23]. In a recent work [24] Sammarruca has applied an isospin-asymmetric EOS derived microscopically from high-precision chiral few-nucleon interactions to study these correlations for a family of mirror pairs.

The nuclear symmetry energy, which is defined as the difference between the energies of pure neutron and symmetric nuclear matter, is an important physical quantity in nuclear physics and astrophysics (see, e.g., [25-27]). It can account for many experimental facts at low nuclear densities, especially the existence of neutron skin. The size of the neutron skin is determined by the relative strengths of the symmetry energy between the central near-saturation and peripheral lessdense regions. Therefore the neutron-skin thickness is a measure of the density dependence of the symmetry energy around saturation [16,28-30]. We have investigated possible relationships between the neutron-skin thickness of spherical [31] and deformed [12] neutron-rich nuclei and the symmetry energy characteristics of nuclear matter for these nuclei. In Refs. [12,31] the analysis of the nuclear symmetry energy, the neutron pressure, and the asymmetric compressibility has been carried out on the basis of the Brueckner EDF for infinite nuclear matter. The capability of the coherent density fluctuation model (CDFM) [32,33] to provide a transparent and analytic way for the transition from nuclear matter to finite nuclei has been demonstrated in these studies.

In the present paper we aim to investigate the relations between the quantities mentioned above among isotopic and isotonic chains with different masses. We focus on nuclei in the mass region $A=48-60$, in which the Ni isotopes and respective mirror nuclei are studied. As an important task, we search for possible correlations between the neutron-skin thickness and the EOS parameters (symmetry energy, pressure, asymmetric compressibility) for various Ni isotopes and their corresponding mirror partners. For the case of these isotopes such correlations have already being investigated in Refs. [12,31,34]. Here we also calculate the proton skins of Argon isotopes $(A=32-40)$ and predictions for them are made, in comparison with the empirical data [35] and the microscopic results of Sammarruca [24]. Also, we inspect the relation between the neutron skin and the difference of the proton radii of the corresponding mirror nuclei for the $Z=20$ and $Z=28$ isotopic chains and for $N=14$ and $N=50$ isotonic chains. Finally, we pay particular attention to the $Z=20$ isotopic chain, including ${ }^{48} \mathrm{Ca}$, inspired by the new experiment on this nucleus (CREX) that is ongoing at JLAB [14].

The nuclear densities and radii are calculated within a self-consistent Hartree-Fock-Bogoliubov (HFB) method by using the cylindrical transformed deformed harmonic-oscillator basis (HFBTHO) [36,37] that has been adopted previously in Refs. [38,39]. The results for the symmetry energy and related quantities in the specified nuclei are obtained in the CDFM framework by use of Brueckner and Skyrme EDFs for infinite nuclear matter with two Skyrme-type effective interactions: SLy4 and SkM*. 
The structure of this paper is the following. In Section 2 we present the relation between the neutron skin of a nucleus and the difference between the proton radii of the corresponding mirror nuclei in the presence of perfect charge symmetry. Section 3 contains the definitions of the key EOS parameters in nuclear matter and CDFM formalism that provides a way to calculate the intrinsic quantities in finite nuclei. The numerical results and discussions are presented in Section 4. The summary of the results and main conclusions of the study are given in Section 5.

\section{Skins of mirror nuclei}

Mirror nuclei, with interchanged numbers of protons and neutrons, are expected to have similar nuclear structure due to the isospin symmetry of nuclear forces. For instance, the level schemes of mirror nuclei should be identical if isospin symmetry is fully exact. Of course, some differences appear due to the fact that the Coulomb interaction breaks the isospin symmetry.

Usually the neutron-skin thickness is associated with the difference between the neutron and proton rms radii (we note that in our previous study [40] the formation of a neutron skin in eveneven isotopes of $\mathrm{Ni}, \mathrm{Kr}$, and $\mathrm{Sn}$ was analyzed in terms of various definitions). The neutron- and proton-skin thicknesses are, correspondingly:

$$
\Delta R_{n}=R_{n}(Z, N)-R_{p}(Z, N)
$$

and

$$
\Delta R_{p}=R_{p}(Z, N)-R_{n}(Z, N),
$$

where $R_{n}(Z, N)$ and $R_{p}(Z, N)$ are the rms radii of the neutron and proton density distributions with $Z$ protons and $N$ neutrons. As can be seen

$$
\Delta R_{n}=-\Delta R_{p}
$$

Under the assumption of exact charge symmetry, the neutron radius of a given nucleus is identical to the proton radius of its mirror nucleus:

$$
R_{n}(Z, N)=R_{p}(N, Z) .
$$

Let us introduce the following difference of the proton radii of a given nucleus and its mirror one:

$$
\Delta R_{\text {mirr }}=R_{p}(N, Z)-R_{p}(Z, N)
$$

Thus, in the case of the exact charge symmetry, using Eq. (4) in Eq. (1), one can obtain the equality of $\Delta R_{n}$ and $\Delta R_{\text {mirr }}$ :

$$
\Delta R_{n}=R_{p}(N, Z)-R_{p}(Z, N)=\Delta R_{\text {mirr }} .
$$

Hence, from accurate measure of the charge radii of the mirror pair nuclei, $(Z, N)$ and $(N, Z)$, the neutron skin thickness of the $(Z, N)$ nucleus can be obtained, provided Coulomb effects are properly taken into account in the data analyses. This could be an alternative to the existing methods to determine neutron and proton skins. 


\section{Nuclear EOS parameters in nuclear matter and finite nuclei}

The nuclear matter EOS is conventionally defined as the binding energy per nucleon and can be approximately expressed as

$$
E(\rho, \delta)=E(\rho, 0)+S^{A N M}(\rho) \delta^{2}+O\left(\delta^{4}\right)+\cdots
$$

in terms of the isospin asymmetry $\delta=\left(\rho_{n}-\rho_{p}\right) / \rho\left(\rho, \rho_{n}\right.$ and $\rho_{p}$ being the baryon, neutron and proton densities, respectively) (see, e.g., [12,31,41,42]). In Eq. (7) $E(\rho, 0)$ is the energy of isospin-symmetric matter. Near the saturation density $\rho_{0}$ the symmetry energy for ANM, $S^{A N M}(\rho)$, can be expanded as

$$
S^{A N M}(\rho)=\left.\frac{1}{2} \frac{\partial^{2} E(\rho, \delta)}{\partial \delta^{2}}\right|_{\delta=0}=a_{4}+\frac{p_{0}^{A N M}}{\rho_{0}^{2}}\left(\rho-\rho_{0}\right)+\frac{\Delta K^{A N M}}{18 \rho_{0}^{2}}\left(\rho-\rho_{0}\right)^{2}+\cdots .
$$

The parameter $a_{4}$ is the symmetry energy at equilibrium $\left(\rho=\rho_{0}\right)$. The pressure $p_{0}^{A N M}$

$$
p_{0}^{A N M}=\left.\rho_{0}^{2} \frac{\partial S^{A N M}}{\partial \rho}\right|_{\rho=\rho_{0}}
$$

and the curvature $\triangle K^{A N M}$

$$
\Delta K^{A N M}=\left.9 \rho_{0}^{2} \frac{\partial^{2} S^{A N M}}{\partial \rho^{2}}\right|_{\rho=\rho_{0}}
$$

of the nuclear symmetry energy at $\rho_{0}$ govern its density dependence and thus provide important information on the properties of the nuclear symmetry energy at both high and low densities. The widely used "slope" parameter $L^{A N M}$ is related to the pressure $p_{0}^{A N M}$ [Eq. (9)] by

$$
L^{A N M}=\frac{3 p_{0}^{A N M}}{\rho_{0}} .
$$

In our previous works $[12,31,43,44]$, as well as in the present paper, the transition from the properties of nuclear matter to those of finite nuclei has been made using the CDFM. The model is a natural extension of the Fermi-gas one. It is based on the $\delta$-function approximation of the generator coordinate method [45] and includes nucleon-nucleon correlations of collective type. In the present work CDFM is applied to our studies of the symmetry energy and related quantities. In the CDFM the one-body density matrix $\rho\left(\mathbf{r}, \mathbf{r}^{\prime}\right)$ is a coherent superposition of the one-body density matrices $\rho_{x}^{N M}\left(\mathbf{r}, \mathbf{r}^{\prime}\right)$ for spherical "pieces" of nuclear matter ("fluctons") with densities $\rho_{x}(\mathbf{r})=\rho_{0}(x) \Theta(x-|\mathbf{r}|)$ and $\rho_{0}(x)=3 A / 4 \pi x^{3}$. It has the form:

$$
\rho\left(\mathbf{r}, \mathbf{r}^{\prime}\right)=\int_{0}^{\infty} d x|\mathcal{F}(x)|^{2} \rho_{x}^{N M}\left(\mathbf{r}, \mathbf{r}^{\prime}\right)
$$

with

$$
\rho_{x}^{N M}\left(\mathbf{r}, \mathbf{r}^{\prime}\right)=3 \rho_{0}(x) \frac{j_{1}\left(k_{F}(x)\left|\mathbf{r}-\mathbf{r}^{\prime}\right|\right)}{\left(k_{F}(x)\left|\mathbf{r}-\mathbf{r}^{\prime}\right|\right)} \Theta\left(x-\frac{\left|\mathbf{r}+\mathbf{r}^{\prime}\right|}{2}\right) .
$$

In (13) $j_{1}$ is the first-order spherical Bessel function and

$$
k_{F}(x)=\left(\frac{3 \pi^{2}}{2} \rho_{0}(x)\right)^{1 / 3} \equiv \frac{\beta}{x}
$$


with

$$
\beta=\left(\frac{9 \pi A}{8}\right)^{1 / 3} \simeq 1.52 A^{1 / 3}
$$

is the Fermi momentum of the nucleons in the flucton with a radius $x$. It follows from Eqs. (12) and (13) that the density distribution in the CDFM has the form:

$$
\rho(\mathbf{r})=\int_{0}^{\infty} d x|\mathcal{F}(x)|^{2} \rho_{0}(x) \Theta(x-|\mathbf{r}|)
$$

and from (16) that in the case of monotonically decreasing local density $(d \rho / d r \leq 0)$ the weight function $|\mathcal{F}(x)|^{2}$ can be obtained from a known density (theoretically or experimentally obtained):

$$
|\mathcal{F}(x)|^{2}=-\left.\frac{1}{\rho_{0}(x)} \frac{d \rho(r)}{d r}\right|_{r=x}
$$

with normalization

$$
\int_{0}^{\infty} d x|\mathcal{F}(x)|^{2}=1 .
$$

Following the CDFM scheme, the symmetry energy, the slope, and the curvature for finite nuclei can be defined weighting these quantities for nuclear matter by means of the weight function $|\mathcal{F}(x)|^{2}$ [Eq. (17)]. They have the following forms (see, for instance, Ref. [31]):

$$
\begin{aligned}
& S(A)=\int_{0}^{\infty} d x|\mathcal{F}(x)|^{2} S^{A N M}(x), \\
& p_{0}(A)=\int_{0}^{\infty} d x|\mathcal{F}(x)|^{2} p_{0}^{A N M}(x), \\
& \Delta K(A)=\int_{0}^{\infty} d x|\mathcal{F}(x)|^{2} \Delta K^{A N M}(x) .
\end{aligned}
$$

Analytical expressions for the nuclear matter quantities $S^{A N M}(x), p_{0}^{A N M}(x)$, and $\Delta K^{A N M}(x)$ [Eqs. (19)-(21)] derived on the basis of Brueckner EDF can be found in Ref. [31]. As it was mentioned before, results for the isotopic and isotonic evolution of the symmetry energy obtained also by Skyrme EDF will be presented.

As far as the densities and the rms radii of the mirror nuclei are concerned, they have been obtained within the Skyrme HFB method. The HFBTHO code [36,37] solves the nuclear Skyrme HFB problem by using the cylindrical transformed deformed harmonic-oscillator basis. In our case we perform spherical calculations taking the cylindrical basis in its spherical limit. In HFBTHO, the direct term of the Coulomb potential to the total energy is taken into account. 
Table 1

Neutron $\left(R_{n}\right)$, proton $\left(R_{p}\right)$, matter $\left(R_{m}\right)$, and charge $\left(R_{c}\right)$ rms radii (in fm) calculated with SLy4 force for $Z=10$ and $Z=18$ isotopic chains. The proton skins $\Delta R_{p}$ [Eq. (2)] (in fm) are also shown in comparison with the results obtained in Ref. [24].

\begin{tabular}{lllllllll}
\hline Nucleus & $Z$ & $N$ & $R_{n}$ & $R_{p}$ & $R_{m}$ & $R_{c}$ & $\Delta R_{p}$ & $\Delta R_{p}[24]$ \\
\hline${ }^{16} \mathrm{Ne}$ & 10 & 6 & 2.51 & 2.89 & 2.76 & 3.00 & 0.378 & $0.422 \pm 0.022$ \\
${ }^{18} \mathrm{Ne}$ & & 8 & 2.67 & 2.85 & 2.77 & 2.96 & 0.175 & $0.186 \pm 0.012$ \\
${ }^{20} \mathrm{Ne}$ & & 10 & 2.81 & 2.84 & 2.82 & 2.95 & 0.029 & $0.032 \pm 0.006$ \\
${ }^{30} \mathrm{Ar}$ & 18 & 12 & 3.00 & 3.32 & 3.20 & 3.42 & 0.323 & $0.352 \pm 0.019$ \\
${ }^{32} \mathrm{Ar}$ & & 14 & 3.07 & 3.28 & 3.19 & 3.38 & 0.216 & $0.225 \pm 0.013$ \\
${ }^{34} \mathrm{Ar}$ & & 16 & 3.17 & 3.29 & 3.23 & 3.39 & 0.123 & $0.127 \pm 0.012$ \\
${ }^{36} \mathrm{Ar}$ & & 18 & 3.26 & 3.31 & 3.29 & 3.40 & 0.046 & $0.046 \pm 0.007$ \\
\hline
\end{tabular}

\section{Results of calculations and discussion}

\subsection{Predictions for nuclear skins. Results of the relation between the neutron skin of a nucleus and the difference between the proton radii of the mirror pair}

We show first in Table 1 the results for the rms radii and proton skins predictions for $Z=10$ and $Z=18$ isotopic chains including neutron-deficient even-even isotopes in each chain. The values of these characteristics are given for the case of the SLy4 effective force. It can be seen that for the neutron-deficient $\mathrm{Ne}$ and Ar nuclei the neutron rms radius is much smaller than the corresponding charge radius. This has the obvious implication that the neutron-deficient nuclei possess an extended proton skin, which is clearly demonstrated in Table 1. A good agreement between our results and the relativistic mean-field (RMF) calculations [46] is achieved for $R_{n}$ and $R_{c}$, as well as with the RMF predictions for $\Delta R_{n}=-\Delta R_{p}$ [Eq. (3)] in [47] and the relativistic Hartree-Bogoliubov model results [48]. There is also a good agreement of the proton skin values derived from the HFBTHO code with the proton skins obtained in Ref. [24] on the base of EOS with high-precision chiral forces. In particular, the obtained proton skins of the less neutron-deficient $\mathrm{Ne}$ and $\mathrm{Ar}$ isotopes are in the interval covering the estimated theoretical errors in Ref. [24]. The latter include uncertainties due to the variations of the cutoff parameter and the chosen many-body method to calculate the skins.

In Table 2 we list the values of the proton skins obtained with $\mathrm{SkM}^{*}$ force for the same isotopes considered in Table 1. Also shown in Table 2 are the neutron-skin thickness of the corresponding mirror partners, as well as the proton radii difference of the mirror pair $\Delta R_{\text {mirr }}$. Indeed, the neutron skins of the corresponding neutron-rich mirror nuclei are smaller than the proton skins for comparable values of proton-neutron asymmetry. For a given proton excess $(Z-N)$ the corresponding proton-skin thickness is observed to be larger than the neutron-skin thickness for the same value of neutron excess $(N-Z)$. This is obviously due to the Coulomb repulsion of protons. We note that the obtained $\Delta R_{n}$ value of ${ }^{16} \mathrm{Ne}$ is comparable with the value of $0.333 \pm 0.016$ from Ref. [24]. It can be seen from Tables 1 and 2 that a small difference is observed between the values of the predicted proton skins $\Delta R_{p}$ for the considered $Z=10$ and $Z=18$ nuclei in respect to the Skyrme force used in the calculations. In particular, the values of $\Delta R_{p}$ obtained with SLy4 force are larger than those with $\mathrm{SkM}^{*}$ force in the case of $\mathrm{Ne}$ isotopes, while they are smaller in the case of Ar isotopes.

Next, we show in Fig. 1 the calculated HFB results for the proton skins of Argon isotopes with $A=29-40$. They are compared with the theoretical predictions from [24] and the experi- 
Table 2

Predicted proton skins $\Delta R_{p}$ [Eq. (2)] (in fm) for the same $Z=$ 10 and $Z=18$ nuclei presented in Table 1 (columns 1 and 2), neutron skins $\Delta R_{n}$ [Eq. (1)] (in fm) of the corresponding mirror nuclei (columns 3 and 4), and $\Delta R_{\text {mirr }}$ [Eq. (6)] (in fm) (column 5) calculated with $\mathrm{SkM}^{*}$ force.

\begin{tabular}{lllll}
\hline Nucleus & $\Delta R_{p}$ & Mirror nucleus & $\Delta R_{n}$ & $\Delta R_{\text {mirr }}$ \\
\hline${ }^{16} \mathrm{Ne}$ & 0.366 & ${ }^{16} \mathrm{C}$ & 0.308 & -0.360 \\
${ }^{18} \mathrm{Ne}$ & 0.160 & ${ }^{18} \mathrm{O}$ & 0.111 & -0.147 \\
${ }^{20} \mathrm{Ne}$ & 0.024 & ${ }^{20} \mathrm{Ne}$ & -0.024 & 0.000 \\
${ }^{30} \mathrm{Ar}$ & 0.327 & ${ }^{30} \mathrm{Mg}$ & 0.238 & -0.308 \\
${ }^{32} \mathrm{Ar}$ & 0.222 & ${ }^{32} \mathrm{Si}$ & 0.136 & -0.188 \\
${ }^{34} \mathrm{Ar}$ & 0.128 & ${ }^{34} \mathrm{~S}$ & 0.041 & -0.088 \\
${ }^{36} \mathrm{Ar}$ & 0.044 & ${ }^{36} \mathrm{Ar}$ & -0.044 & 0.000 \\
\hline
\end{tabular}

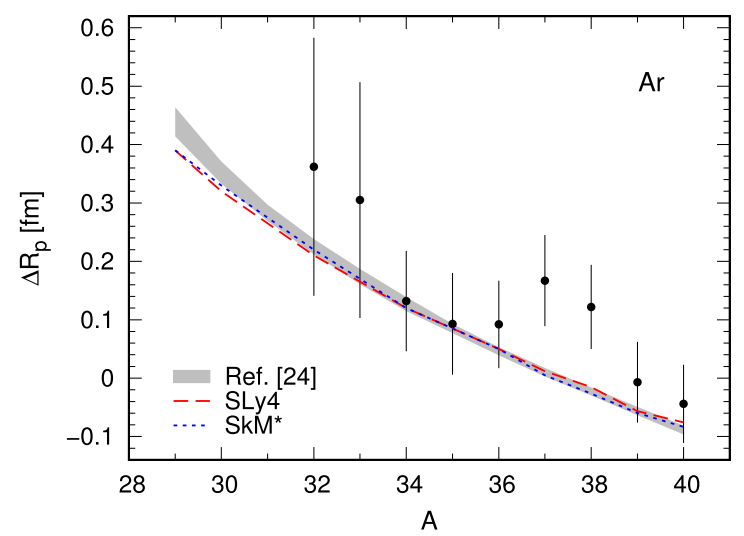

Fig. 1. (Color online.) Predicted proton skins of Ar isotopes as a function of the mass number $A$. The results calculated with SLy4 and SkM* Skyrme interactions are given by red dashed and blue dotted lines, respectively. The gray band represents the result of Sammarruca [24]. The experimental data points are from Ozawa et al. [35].

mental proton-skin thicknesses for isotopes ${ }^{32-40} \mathrm{Ar}$ deduced from the interaction cross sections of ${ }^{31-40} \mathrm{Ar}$ on carbon target [35]. In spite of the large errors of the empirical data, the predictions of both theoretical methods describe reasonably well their trend, namely the monotonic decrease of the proton skin with increasing of the neutron number $N$ in a given isotopic chain. Actually, the data in Fig. 1 cover a range of $N$ that includes the magic number $N=20$, but an enhancement of the proton skin is seen earlier at $N=19$. It was pointed out in Ref. [49] that in the case of Argon isotopes $N=20$ was not found to be a magic number. Most likely this is due to the inversion of the standard $s d$-shell configuration and the intruder $f p$-shell, as it has been proved for the neutron-rich ${ }^{32} \mathrm{Mg}$ nucleus, which lies in the much explored island of inversion at $N=20$ (see, for instance, Ref. [34]). Obviously, more detailed consideration of the Ar isotopes around $A=38$ is necessary to give a clear answer about the role of the shell effects on the behavior of the proton skin data in this mass region.

The relation between the neutron skin $\Delta R_{n}$ and the proton radii difference of the mirror pair $\Delta R_{\text {mirr }}$ as defined in Eq. (6) is presented in Fig. 2 on the examples of $Z=20$ and $Z=28$ isotopic chains and two isotonic chains with $N=14$ and $N=50$. In all four cases a linear relation 

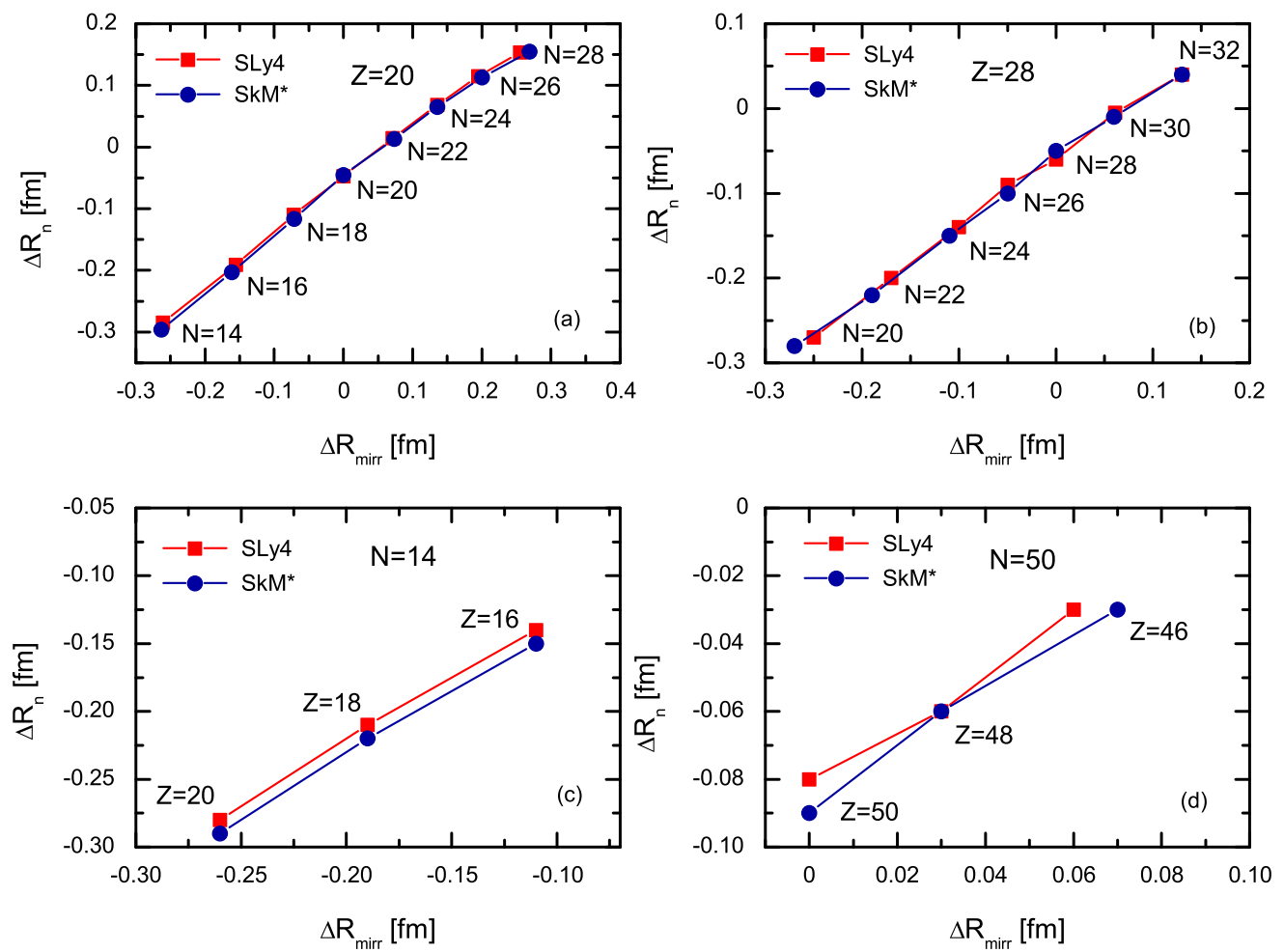

Fig. 2. (Color online.) Relation between the neutron skin $\Delta R_{n}$ and $\Delta R_{\text {mirr }}$ [Eq. (6)] for the $Z=20$ (a) and $Z=28$ (b) isotopic chains and for the $\mathrm{N}=14$ (c) and $\mathrm{N}=50$ (d) isotonic chains. The results with SLy4 and SkM* forces are given by red (with squares) and blue (with dots) lines, respectively.

between these characteristics is observed. Note that the results are obtained in the presence of Coulomb effects. It is seen from Fig. 2 that both Skyrme interactions provide similar results. To explore to which extent the linear relation between $\Delta R_{n}$ and $\Delta R_{\text {mirr }}$

$$
\Delta R_{n}=c\left(\Delta R_{\text {mirr }}\right)+d
$$

holds based on the four chains, we perform a linear fit of the curves corresponding to SkM* and SLy4 forces. The parameters of the generalized linear relation are the following:

$$
c=0.866 \pm 0.037, \quad d=-0.0633 \pm 0.0041
$$

in the case of $\mathrm{SkM}^{*}$ force and

$$
c=0.862 \pm 0.042, \quad d=-0.0575 \pm 0.0041
$$

in the case of SLy4 force. We would like to note that the exhibited linear relation does not depend much on the effective Skyrme interaction used in the calculations. Moreover, based on microscopic EOS similar linear relations are shown in Ref. [24] for chains with $N=28, Z=10$, and $Z=20$. Confirming a global relation between $\Delta R_{n}$ and $\Delta R_{\text {mirr }}$ regardless $Z$ and $N$, the two different theoretical methods used in the present work and in Ref. [24] yield similar predictions of the linear relation (see Eq. (11) in [24]). Thus, measuring the proton radii of the mirror pair one can get an access to the neutron-skin thickness of the $(Z, N)$ nucleus [Eqs. (22)-(24)]. 
Here we would like to stress that the linear fit performed in the present calculations follows the line in Ref. [24], where a family of mirror pairs is considered to determine the relation between $\Delta R_{n}$ and $\Delta R_{\text {mirr }}$ for a single interaction, while in Brown [22] this correlation is determined for a particular mirror pair $\left({ }^{52} \mathrm{Ni}-{ }^{52} \mathrm{Cr}\right)$ using 48 different Skyrme functionals. Also, as was pointed out in Ref. [24], Eqs. (22)-(24) give a small negative value of $\Delta R_{n}$ in the limit of $\Delta R_{\text {mirr }}=$ $R_{p}(N, Z)-R_{p}(Z, N) \rightarrow 0$, which makes sense in the light of Coulomb effects. Hence, Eq. (6) suggests appropriate modifications to account for Coulomb effects including the slope $c<1$. As a result, a small deviation from the obtained linear fit of the relation (22) can be observed for the case of the mass region of heavier $N=50$ isotones [given in Fig. 2(d)].

\subsection{Symmetry energy of nuclei from isotopic chains with $Z=20$ and $Z=28$ and isotonic chains with $N=14$ and $N=50$ and their respective mirror partner nuclei}

In what follows, we show our results for the symmetry energy $S$ obtained within the CDFM using first the Brueckner EDF for the symmetry energy in infinite nuclear matter $S^{A N M}$ in Eq. (19), while the weight function $|\mathcal{F}(x)|^{2}$ is obtained using Eq. (17) by means of the density distribution within the Skyrme HFB method (the HFBTHO densities). Second, we calculate in the CDFM the symmetry energy $S$ using also the Skyrme EDF. In this case there is a selfconsistency between the way to obtain $|\mathcal{F}(x)|^{2}$ in the Skyrme HFB method and the use of the Skyrme EDF to obtain the symmetry energy. Also, we inspect the correlation of the neutron-skin thickness $\Delta R_{n}$ of nuclei in a given isotopic chain with the $S$ [Eq. (19)], $p_{0}$ [Eq. (20)], and $\Delta K$ [Eq. (21)] parameters extracted from the density dependence of the symmetry energy around the saturation density. Complementary to the analyses in Refs. [12,31], in the present work we put emphasis on the possible existence of such correlations for the chain of mirror partners of the corresponding isotopes, which is one of the main tasks of the present work.

The mass dependence of the symmetry energy $S$ for the Ni isotopes $(Z=28)$ with $A=$ $48-60$ and their mirror nuclei by using the Brueckner and Skyrme EDFs with SLy4 and SkM* forces is presented in Fig. 3. The behavior of the curves for the two functionals is similar and the values of $S$ obtained with Skyrme EDF with SLy4 force are larger in comparison with the corresponding values deduced in other cases. An important result, which can be seen from Fig. 3, is that the mirror partner nuclei show the same linear behavior observed in the evolution of the symmetry energy in Ni chain (see also Ref. [31]) containing an inflection point ("kink") at the double-magic ${ }^{56} \mathrm{Ni}$ nucleus. We note that there is a small shift of the curve for mirror partners with respect to the curve for the Ni isotopes with a smooth growing deviation between them with increasing $|N-Z|$.

The results for the correlation between the neutron (proton)-skin thickness and the symmetry energy, the pressure, and the asymmetric compressibility for the Ni isotopes and their mirror partners calculated on the basis of the Brueckner EDF for ANM and using SLy4 force are shown in Fig. 4. It is seen from the figure that there exists an approximate linear correlation between $\Delta R_{n}$ and $S$ not only for the even-even Ni isotopes with $A=48-60$, but also for the mirror nuclei between $\Delta R_{p}=-\Delta R_{n}$ and $S$. We observe a smooth growth of the symmetry energy till the double-magic nucleus ${ }^{56} \mathrm{Ni}$ and then a linear decrease of $S$ while the neutron-skin thickness of the isotopes increases. This tendency happens also for the chain of the mirror nuclei from ${ }^{48} \mathrm{Ca}$ to ${ }^{60} \mathrm{Ge}$ with an inflection point transition at the double-magic ${ }^{56} \mathrm{Ni}$ nucleus. The correlation between $\Delta R_{n}$ and $p_{0}$ is similar, while a less strong correlation between $\Delta R_{n}$ and $\Delta K$ is found. Here we note that the determined values of the neutron-skin thickness $\Delta R_{n}$ of ${ }^{48} \mathrm{Ca}$ nucleus are $0.155 \mathrm{fm}$ in the case of SkM* and $0.154 \mathrm{fm}$ in the case of SLy4 forces, respectively, in consistency 

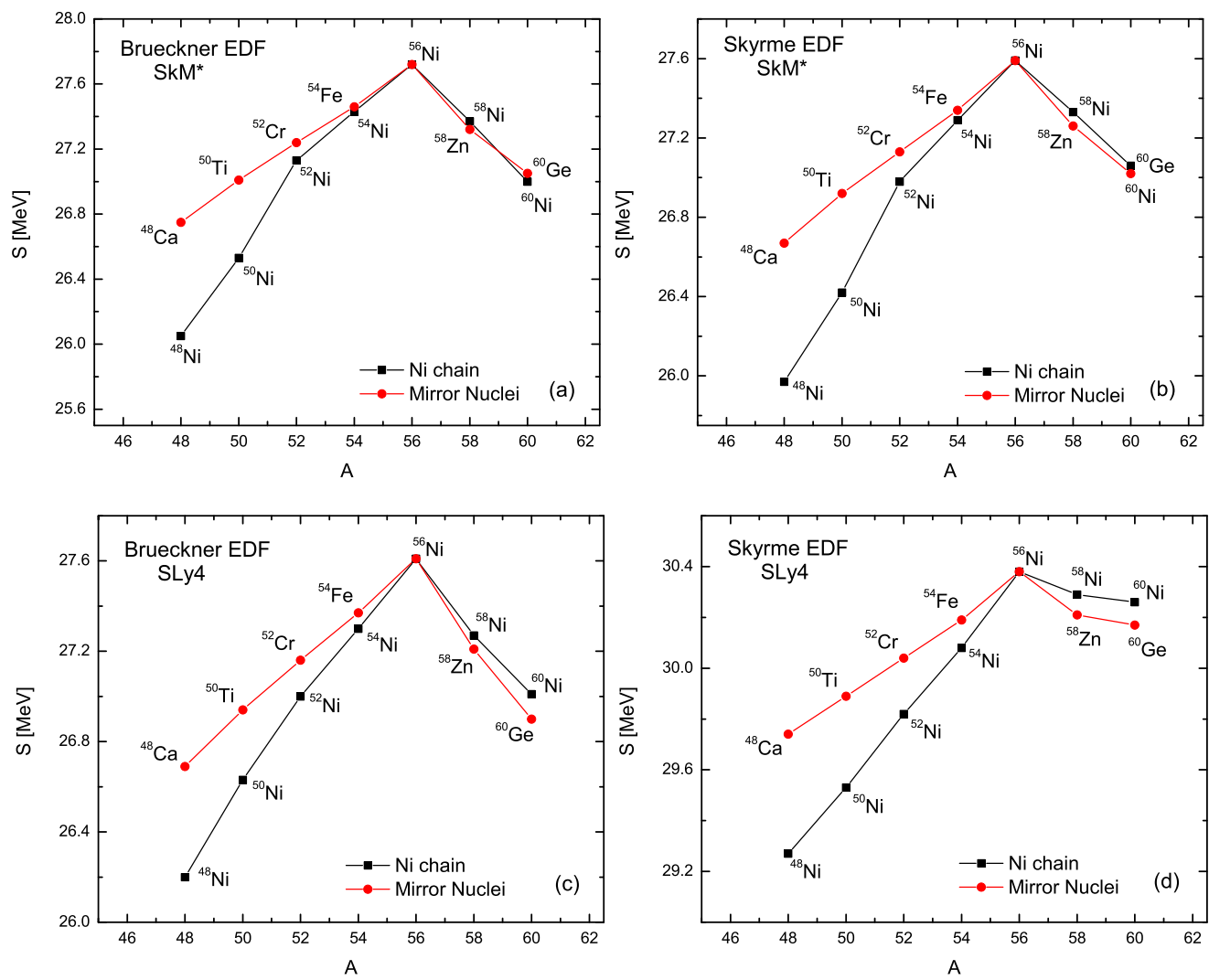

Fig. 3. (Color online.) The symmetry energy $S$ as a function of the mass number $A$ for Ni isotopes (black line with squares) and their mirror nuclei (red line with circles) calculated with Brueckner [panels (a) and (c)] and Skyrme [panels (b) and (d)] EDFs in the case of SkM* and SLy4 forces, respectively.

with the predicted value of $0.159 \mathrm{fm}$ using the Gogny-D1S HFB method [50]. These values are also in good agreement with $a b$ initio calculations based on the chiral effective field theory that yield values between $0.14 \mathrm{fm}$ and $0.20 \mathrm{fm}$ for the neutron skin of ${ }^{48} \mathrm{Ca}$ (see, for instance, the value of $0.181 \pm 0.010$ in Ref. [24]).

In Fig. 5 we display results on the isotopic evolution of the symmetry energy for the $Z=20$ isotopes $(A=34-48)$ and their corresponding mirror partners, extending the latter to include two more isotones with $Z=10$ and 12. They are shown for the cases of Brueckner and Skyrme EDFs with both SkM* and SLy4 forces. This analysis is motivated by the active study of Ca isotopes in the proximity of ${ }^{48} \mathrm{Ca}$, with emphasis on the evolution of the charge radii at both proton-rich [51] and neutron-rich [52] sides. As seen in Fig. 5, our results for the symmetry energy $S$ exhibit a characteristic monotonic dependence on $A$ for $A>36$, in accordance with the predictions for the charge radii of the $\mathrm{Ca}$ isotopes calculated within the HFB method by using of the Skyrme parametrization SV-min [51]. In contrast to the Ni chain presented in Fig. 3 where a "kink" exists at the double-magic ${ }^{56} \mathrm{Ni}$, no "kink" is observed, particularly at the double-magic ${ }^{40} \mathrm{Ca}$ nucleus. However, there is a "kink" at ${ }^{36} \mathrm{Ca}$ that is more pronounced when $\mathrm{SkM}^{*}$ force is used in the calculations with both Brueckner and Skyrme EDFs. The corresponding mirror 

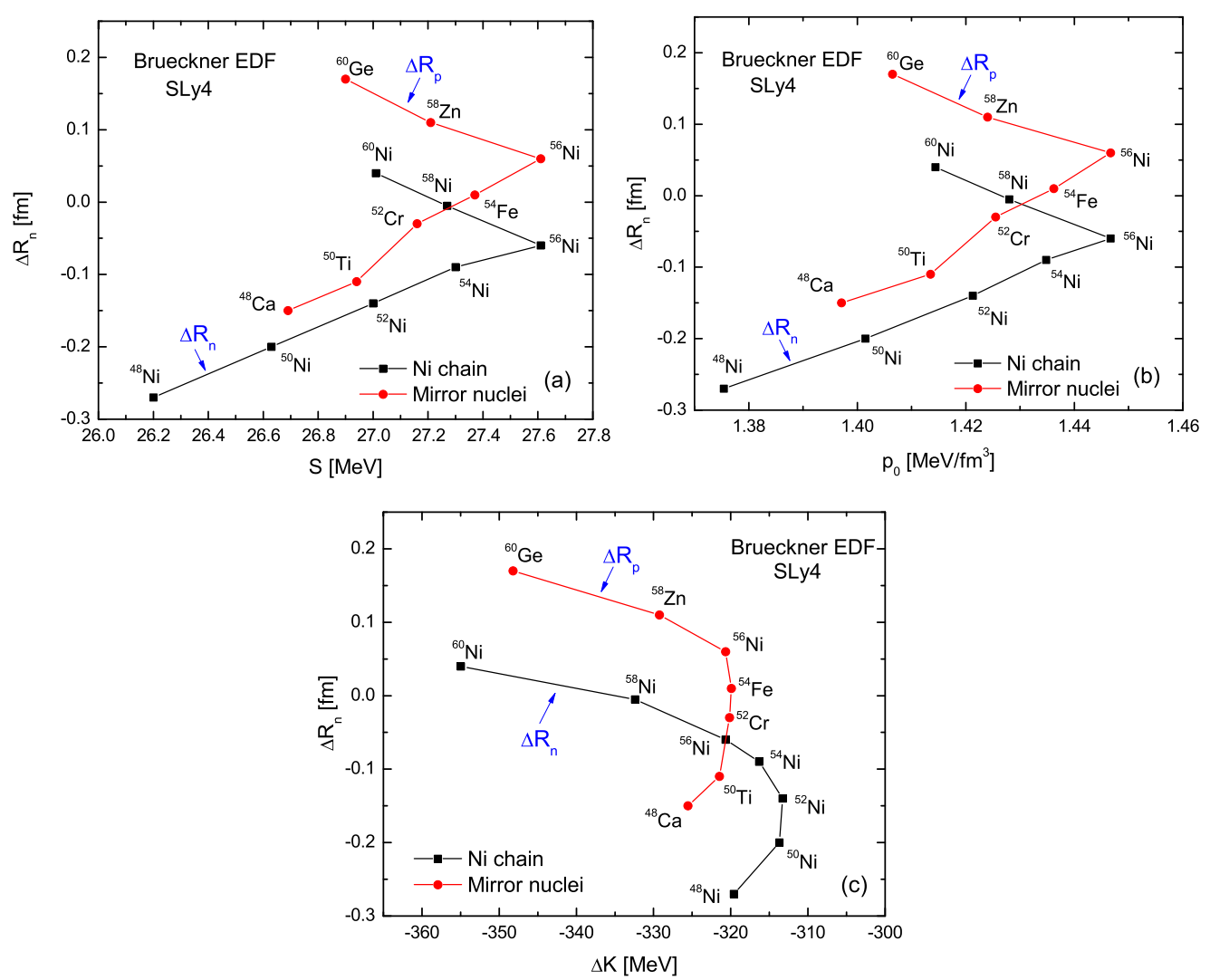

Fig. 4. (Color online.) HFB neutron-skin thickness $\Delta R_{n}$ for Ni isotopes (black line with squares) and corresponding proton skins $\Delta R_{p}=-\Delta R_{n}$ for their mirror nuclei (red line with circles) as a function of the symmetry energy $S$ (a), pressure $p_{0}(\mathrm{~b})$, and asymmetric compressibility $\Delta K$ (c) calculated with Brueckner EDF and SLy4 force.

partners ( $N=20$ isotones) also reveal a linear behavior but with an inflection point transition at ${ }^{34} \mathrm{Si}$ nucleus that is more pronounced with SLy4 force.

The isotopic (isotonic) evolution of the symmetry energy of nuclei with $Z(N)=20$ shown in Fig. 5 requires a deeper analysis. The existence of kinks in the symmetry energy behavior in these chains at ${ }^{36} \mathrm{Ca}$ and ${ }^{34} \mathrm{Si}$ (mostly pronounced with the use of SLy4 interaction) can be attributed to the specific nuclear shell structure in this mass region. Based on the evolution of neutron separation energies and cross sections in light nuclei, it was originally proposed $N=16$ to be a new magic number lying between the usual $N=8$ and 20 for $\mathrm{N}$, O and F nuclei [53]. It has been observed experimentally [54] that the $N=20$ isotonic chain shows an evidence of two new sub-shell closures for $N=14$ and $N=16$. In fact, ${ }^{34} \mathrm{Si}$ and ${ }^{36} \mathrm{~S}$ reveal the typical features of double-magic nuclei. The predicted double-magicity of ${ }^{34} \mathrm{Si}$ is discussed in [55] but, to our knowledge, no unambiguous evidence has been found yet. Nevertheless, the reported first experimental proof that points to a depletion of the central density of protons in the short-lived nucleus ${ }^{34} \mathrm{Si}$ shown in Ref. [56] is in favor of the double-magicity structure of this nucleus, in which the mixing between normally occupied and valence orbits is very limited. This newly observed $N=14, N=16$ or $Z=14, Z=16$ shell stabilization in $Z(N)=20$ chains, correspondingly, 

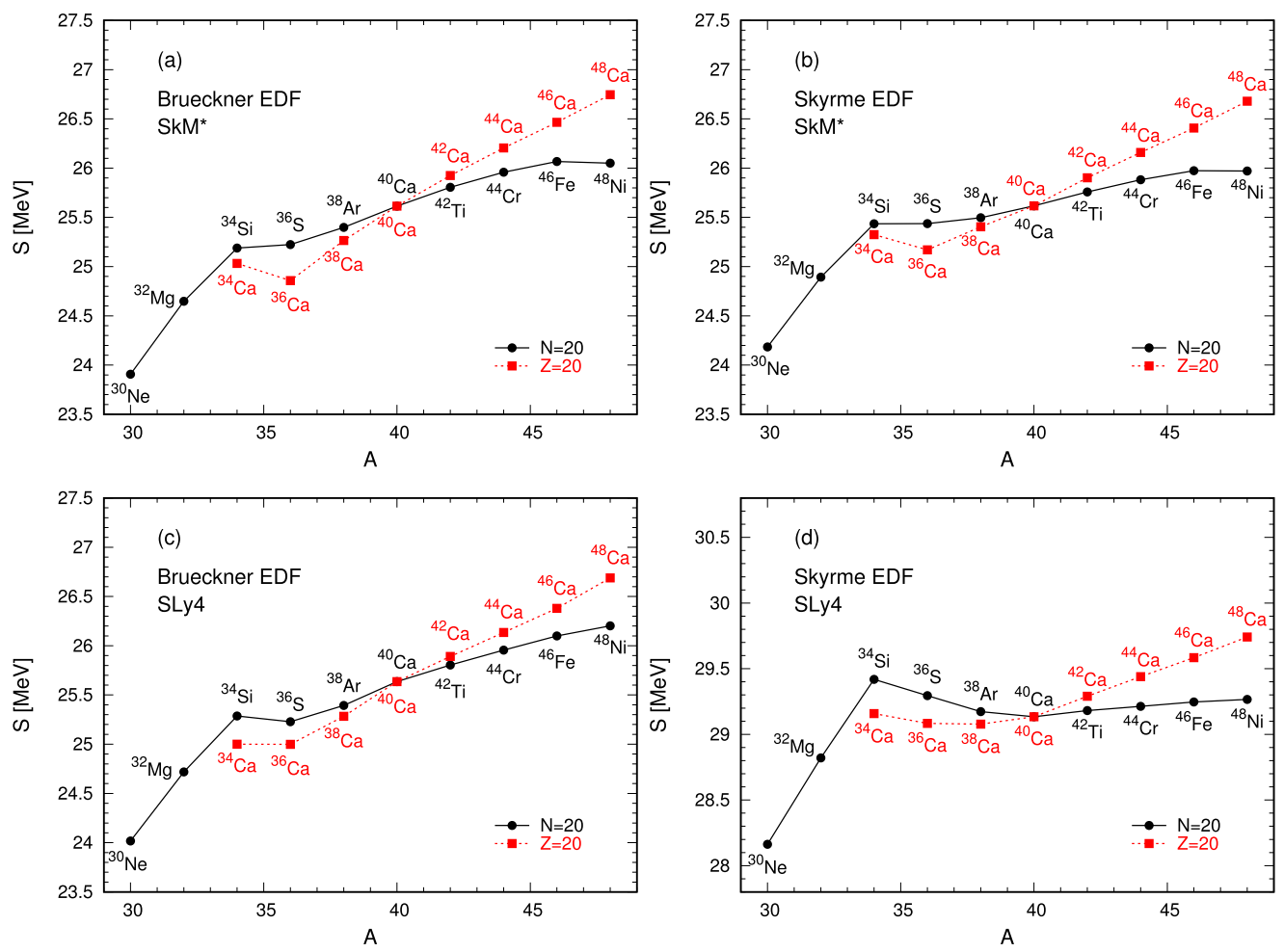

Fig. 5. (Color online.) The symmetry energy $S$ as a function of the mass number $A$ for Ca isotopes (red dotted line with squares) and their mirror nuclei (black solid line with circles) calculated with Brueckner [panels (a) and (c)] and Skyrme [panels (b) and (d)] EDFs in the case of SkM* and SLy4 forces, respectively.

is expected to be symmetric with respect to the isospin projection. Therefore, the ideal region to check such prediction is along the $Z=20 \mathrm{Ca}$ isotopes and their mirror $N=20$ isotones. The detailed spectroscopy of ${ }^{36} \mathrm{Ca}$ and its mirror nucleus ${ }^{36} \mathrm{~S}$ made with RISING at GSI led to the hypothesis of a new "island of inversion" developed by neutron-deficient $\mathrm{Ca}$ isotopes where the onset of inversion may start already at $N=14$ in ${ }^{34} \mathrm{Ca}$ [57].

Fig. 6 illustrates the evolution of the symmetry energy for $N=14$ isotones and their mirror $\mathrm{Si}$ isotopes $(Z=14)$. Our choice of this isotonic chain is motivated by the fact that data for light nuclei, such as those with $N=14$, are likely to be obtained in future electron scattering facilities such as SCRIT and ELISe at FAIR in GSI. It is seen from Fig. 6 that the symmetry energies deduced from SkM* and SLy4 parametrizations vary in the interval 25-30 MeV.

Finally, we show in Fig. 7, in the same manner as in Figs. 3, 5 and 6, the mass dependence of the nuclear symmetry energy $S$ of even-even nuclei from the $N=50$ chain $(A=72-100)$ and their heaviest three mirror Sn isotopes. In this case the need to consider mirror pairs limits the spectrum of realistic possibilities. First, we can observe a strong "kink" at the double-magic ${ }^{78} \mathrm{Ni}$ nucleus along the chain from ${ }^{72} \mathrm{Ti}$ to ${ }^{100} \mathrm{Sn}$, which is a common feature for both EDFs and Skyrme forces used in the calculations. Second, the two curves approach each other going to the double-magic ${ }^{100} \mathrm{Sn}$ nucleus, where they are crossing, similarly to the cases of $Z=28$ and $Z=20$ isotopic chains and their mirror nuclei presented in Figs. 3 and 5, respectively, where the intersection takes place at the double-magic ${ }^{56} \mathrm{Ni}$ and ${ }^{40} \mathrm{Ca}$ nuclei. 

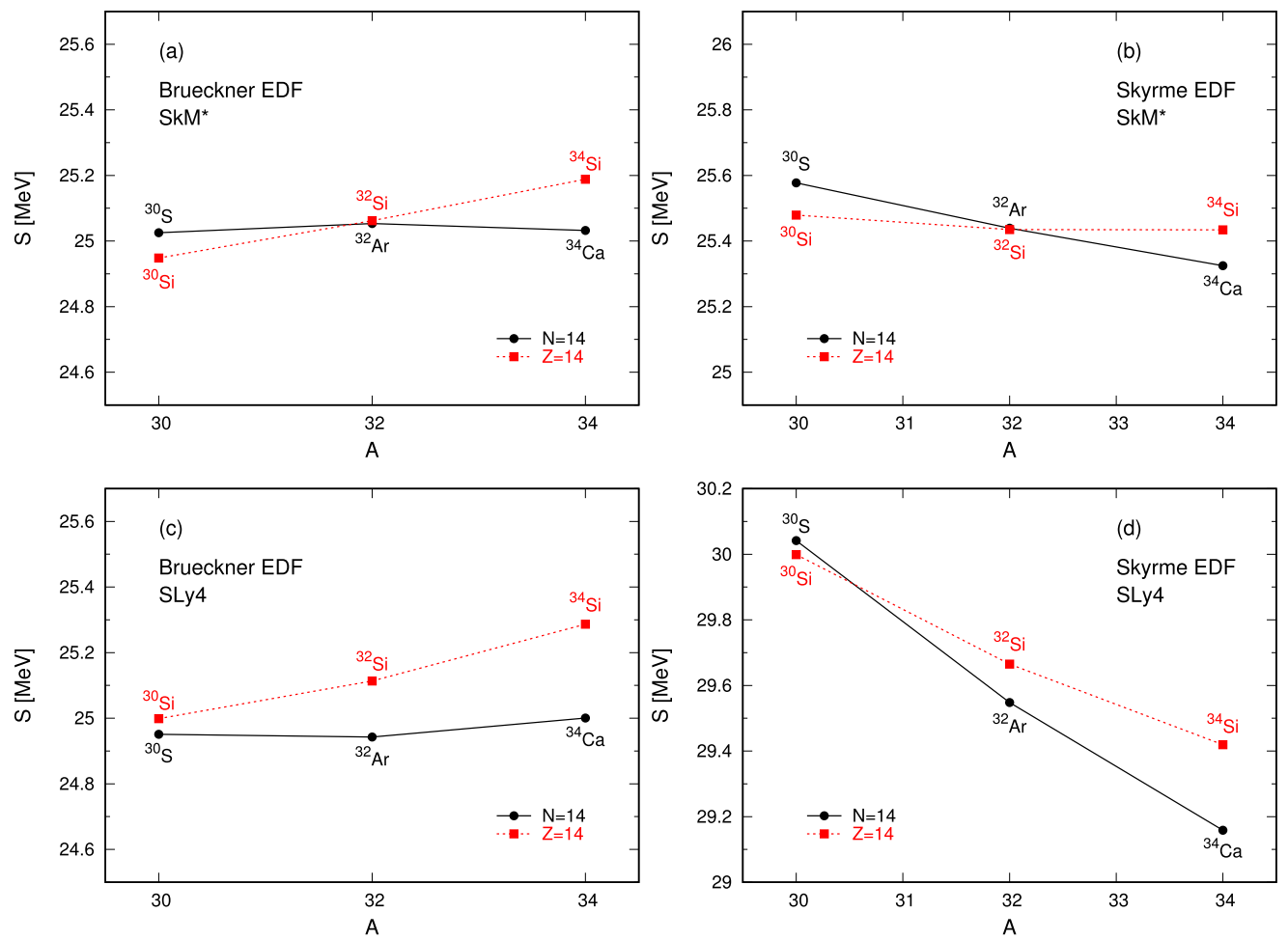

Fig. 6. (Color online.) The symmetry energy $S$ as a function of the mass number $A$ for $N=14$ isotones (black solid line with circles) and their mirror Si isotopes (red dotted line with squares) calculated with Brueckner [panels (a) and (c)] and Skyrme [panels (b) and (d)] EDFs in the case of SkM* and SLy4 forces, respectively.

As a common feature of the results for the nuclear symmetry energy in different chains and their mirror nuclei presented in Figs. 3-7, the calculations with Skyrme EDF using SLy4 force yield larger values of $S$ in comparison with other cases. Similar trend has been found in Ref. [38], where the values of the symmetry energy coefficient $e_{s y m}$ for $\mathrm{Ni}, \mathrm{Sn}$, and $\mathrm{Pb}$ isotopic chains at zero temperature calculated with Skyrme EDF and SLy4 overestimate those obtained with the $\mathrm{SkM}^{*}$ force. The difference in the magnitude of the symmetry energy when using both Skyrme parametrizations is associated with their different saturation properties, namely, for the parameter set SkM* and SLy4, the corresponding symmetry energy of nuclear matter $a_{4}$ at saturation density $\rho_{0}$ is $30.00 \mathrm{MeV}$ and $31.99 \mathrm{MeV}$, respectively (see, for instance, Ref. [58]).

\section{Summary and conclusions}

In this work, the HFB method by using the cylindrical transformed deformed harmonicoscillator basis has been applied to calculations of radii and skins for several mirror pairs in the middle mass range. The existence of an important possible correlation between the neutron (proton) skins and the parameters of the EOS, such as the symmetry energy $S$, the pressure $p_{0}$, and the curvature $\Delta K$, has been investigated. The mentioned EOS parameters have been calculated for Ni isotopic chain with mass number $A=48-60$, as well as for nuclei with $Z=20$, $N=14$, and $N=50$ and their respective mirror partner nuclei, using Brueckner and Skyrme 

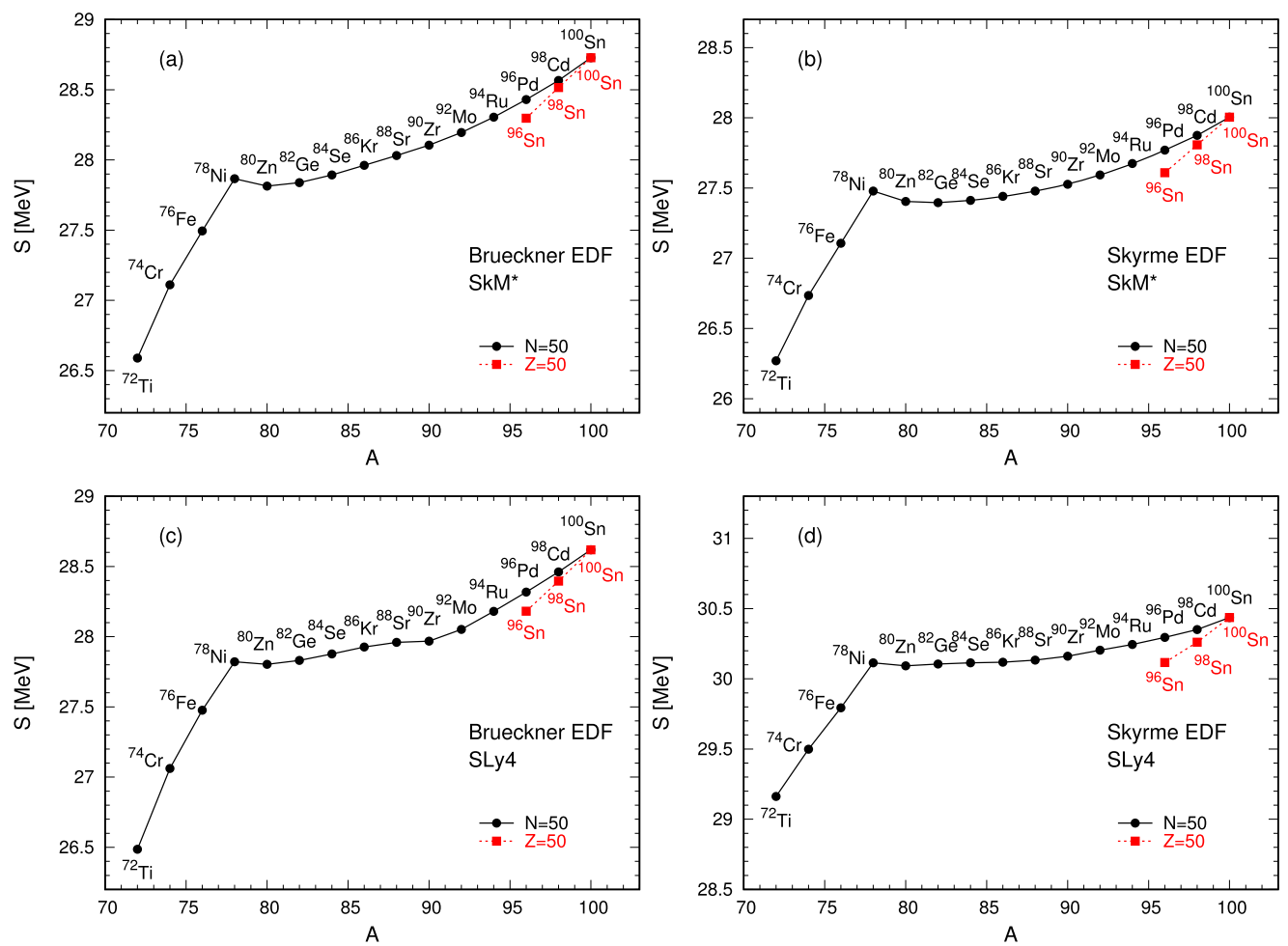

Fig. 7. (Color online.) The symmetry energy $S$ as a function of the mass number $A$ for $N=50$ isotones (black solid line with circles) and their heaviest three mirror $\mathrm{Sn}$ isotopes (red dotted line with squares) calculated with Brueckner [panels (a) and (c)] and Skyrme [panels (b) and (d)] EDFs in the case of SkM* and SLy4 forces, respectively.

EDFs for isospin ANM with two Skyrme-type forces, SkM* and SLy4. The results are obtained within the CDFM that links the properties of nuclear matter with the microscopic description of finite nuclei.

The main results of the present study can be summarized, as follows:

i) Due to Coulomb effects, the predicted proton skins are found larger than the neutron skins of the corresponding mirror partner nuclei. They compare reasonably well with the available empirical data, for instance for Ar isotopes, that are also well described by chiral effective field theory-based EOS;

ii) The studied relation between the neutron skin $\Delta R_{n}$ and the difference between the proton radii $\Delta R_{\text {mirr }}$ for a family of mirror pairs in the presence of Coulomb effects shows clearly a linear dependence. Thus, this appears to be an alternative way to explore neutron skins that may challenge experimentalists to perform high-precision measurements of charge radii of unstable neutron-rich isotopes;

iii) We found in the case of the $\mathrm{Ni}$ isotopic chain and the respective mirror partner nuclei a strong correlation between the neutron (proton)-skin thickness and the symmetry energy $S$ and pressure $p_{0}$ with a "kink" at double-magic ${ }^{56} \mathrm{Ni}$, while the correlation between $\Delta R_{n}\left(\Delta R_{p}\right)$ and the asymmetric compressibility $\Delta K$ is less pronounced. In our opinion, more general 
conclusions for such correlations (including also other nuclear chains of mirror pairs) can be drawn after detailed treatment of nuclear deformation, shell structure and surface effects;

iv) The evolution of the symmetry energy $S$ with the mass number $A$ of nuclei from $Z=20$, $Z=28$, and $N=50$ chains and their mirror nuclei exhibits similar behavior. The curves cross in each chain at the corresponding $N=Z$ nucleus $\left({ }^{40} \mathrm{Ca},{ }^{56} \mathrm{Ni},{ }^{100} \mathrm{Sn}\right)$ and start to deviate from each other with the increase of the level of asymmetry $|N-Z|$.

In principle, studies of elastic and quasi-elastic electron scattering on isotopic and isotonic chains can provide useful information about the evolution of the charge form factors and related charge density distributions, and thus, on the occupation and filling of the single-particle levels of nucleons along the chains (see, e.g., Refs. [59-61]). These investigations being combined with analyses of isospin-dependent properties through the nuclear symmetry energy, its density dependence and related quantities along chains of mirror pairs, may lead to observations of new phenomena related to the proton-to-neutron asymmetry.

\section{CRediT authorship contribution statement}

M.K. Gaidarov: Conceptualization, Methodology, Software, Writing - original draft. I. Moumene: Methodology, Software. A.N. Antonov: Investigation, Methodology, Visualization. D.N. Kadrev: Methodology, Software. P. Sarriguren: Methodology, Writing - review \& editing. E. Moya de Guerra: Methodology, Validation.

\section{Declaration of competing interest}

The authors declare that they have no known competing financial interests or personal relationships that could have appeared to influence the work reported in this paper.

\section{Acknowledgements}

M.K.G., A.N.A., and D.N.K. are grateful for the support of the Bulgarian National Science Fund under Contract No. KP-06-N38/1. P.S. acknowledges support from Ministerio de Ciencia, Innovación y Universidades MCIU/AEI/FEDER, UE (Spain) under Contract No. PGC2018093636-B-I00.

\section{References}

[1] P.-G. Reinhard, W. Nazarewicz, Phys. Rev. C 81 (2010) 051303.

[2] H. Simon, Nucl. Phys. A 787 (2007) 102.

[3] A.N. Antonov, et al., Nucl. Instrum. Methods Phys. Res., Sect. A 637 (2011) 60.

[4] T. Suda, M. Wakasugi, Prog. Part. Nucl. Phys. 55 (2005) 417.

[5] T. Suda, et al., Prog. Theor. Exp. Phys. 2012 (2012) 03 C008.

[6] M. Wakasugi, et al., Nucl. Instrum. Methods Phys. Res., Sect. B 317 (2013) 668.

[7] K. Tsukada, et al., Phys. Rev. Lett. 118 (2017) 262501.

[8] O. Moreno, P. Sarriguren, E. Moya de Guerra, J.M. Udias, T.W. Donnelly, I. Sick, Nucl. Phys. A 828 (2009) 306.

[9] T.W. Donnelly, J. Dubach, I. Sick, Nucl. Phys. A 503 (1989) 589.

[10] http://hallaweb.jlab.org/parity/prex.

[11] S. Abrahamyan, et al., Phys. Rev. Lett. 108 (2012) 112502.

[12] M.K. Gaidarov, A.N. Antonov, P. Sarriguren, E. Moya de Guerra, Phys. Rev. C 85 (2012) 064319.

[13] P.A. Souder, et al., PREX-II: precision parity-violating measurement of the neutron skin of lead, http://hallaweb. jlab.org/parity/prex/, 2011. 
[14] J. Mammei, et al., CREX: parity-violating measurement of the weak charge distribution of ${ }^{48}$ Ca to $0.02 \mathrm{fm}$ accuracy, http://hallaweb.jlab.org/parity/prex/, 2013.

[15] C.J. Horowitz, K.S. Kumar, R. Michaels, Eur. Phys. J. A 50 (2014) 48.

[16] R. Furnstahl, Nucl. Phys. A 706 (2002) 85.

[17] M.H. Mahzoon, M.C. Atkinson, R.J. Charity, W.H. Dickhoff, Phys. Rev. Lett. 119 (2017) 222503.

[18] M.H. Mahzoon, R.J. Charity, W.H. Dickhoff, H. Dussan, S.J. Waldecker, Phys. Rev. Lett. 112 (2014) 162503.

[19] M.C. Atkinson, M.H. Mahzoon, M.A. Keim, B.A. Bordelon, C.D. Pruitt, R.J. Charity, W.H. Dickhoff, Phys. Rev. C 101 (2020) 044303.

[20] C.D. Pruitt, R.J. Charity, L.G. Sobotka, M.C. Atkinson, W.H. Dickhoff, Phys. Rev. Lett. 125 (2020) 102501.

[21] H. Li, H. Xu, Y. Zhou, X. Wang, J. Zhao, L.-W. Chen, F. Wang, arXiv:1910.06170 [nucl-th].

[22] B.A. Brown, Phys. Rev. Lett. 119 (2017) 122502.

[23] J. Yang, J. Piekarewicz, Phys. Rev. C 97 (2018) 014314.

[24] F. Sammarruca, Front. Phys. 6 (2018) 90.

[25] Topical issue on nuclear symmetry energy, Guest editors: Bao-An Li, Angels Ramos, Giuseppe Verde, Isaac Vidaña, Eur. Phys. J. A 50 (2014) 2.

[26] J.M. Lattimer, M. Prakash, Phys. Rep. 442 (2007) 109.

[27] B.A. Li, et al., Phys. Rep. 464 (2008) 113.

[28] S. Typel, B.A. Brown, Phys. Rev. C 64 (2001) 027302.

[29] A. Steiner, M. Prakash, J. Lattimer, P. Ellis, Phys. Rep. 411 (2005) 325.

[30] X. Roca-Maza, M. Centelles, X. Viñas, M. Warda, Phys. Rev. Lett. 106 (2011) 252501.

[31] M.K. Gaidarov, A.N. Antonov, P. Sarriguren, E. Moya de Guerra, Phys. Rev. C 84 (2011) 034316.

[32] A.N. Antonov, V.A. Nikolaev, I.Zh. Petkov, Bulg. J. Phys. 6 (1979) 151;

A.N. Antonov, V.A. Nikolaev, I.Zh. Petkov, Z. Phys. A 297 (1980) 257;

A.N. Antonov, V.A. Nikolaev, I.Zh. Petkov, Z. Phys. A 304 (1982) 239;

A.N. Antonov, V.A. Nikolaev, I.Zh. Petkov, Nuovo Cimento A 86 (1985) 23;

A.N. Antonov, et al., Nuovo Cimento A 102 (1989) 1701;

A.N. Antonov, D.N. Kadrev, P.E. Hodgson, Phys. Rev. C 50 (1994) 164.

[33] A.N. Antonov, P.E. Hodgson, I.Zh. Petkov, Nucleon Momentum and Density Distributions in Nuclei, Clarendon Press, Oxford, 1988;

A.N. Antonov, P.E. Hodgson, I.Zh. Petkov, Nucleon Correlations in Nuclei, Springer-Verlag, Berlin-HeidelbergNew York, 1993.

[34] M.K. Gaidarov, P. Sarriguren, A.N. Antonov, E. Moya de Guerra, Phys. Rev. C 89 (2014) 064301.

[35] A. Ozawa, et al., Nucl. Phys. A 709 (2002) 60.

[36] M.V. Stoitsov, et al., Comput. Phys. Commun. 184 (2013) 1592.

[37] M.V. Stoitsov, J. Dobaczewski, W. Nazarewicz, P. Ring, Comput. Phys. Commun. 167 (2005) 43.

[38] A.N. Antonov, D.N. Kadrev, M.K. Gaidarov, P. Sarriguren, E. Moya de Guerra, Phys. Rev. C 95 (2017) 024314.

[39] A.N. Antonov, D.N. Kadrev, M.K. Gaidarov, P. Sarriguren, E. Moya de Guerra, Phys. Rev. C 98 (2018) 054315.

[40] P. Sarriguren, M.K. Gaidarov, E.M. de Guerra, A.N. Antonov, Phys. Rev. C 76 (2007) 044322.

[41] A.E.L. Dieperink, Y. Dewulf, D. Van Neck, M. Waroquier, V. Rodin, Phys. Rev. C 68 (2003) 064307.

[42] L.-W. Chen, Phys. Rev. C 83 (2011) 044308.

[43] A.N. Antonov, M.K. Gaidarov, P. Sarriguren, E. Moya de Guerra, Phys. Rev. C 94 (2016) 014319.

[44] I.C. Danchev, A.N. Antonov, D.N. Kadrev, M.K. Gaidarov, P. Sarriguren, E. Moya de Guerra, Phys. Rev. C 101 (2020) 064315.

[45] J.J. Griffin, J.A. Wheeler, Phys. Rev. 108 (1957) 311.

[46] G.A. Lalazissis, A.R. Farhan, M.M. Sharma, Nucl. Phys. A 628 (1998) 221.

[47] L.S. Geng, H. Toki, A. Ozawa, J. Meng, Nucl. Phys. A 730 (2004) 80.

[48] G.A. Lalazissis, D. Vretenar, P. Ring, Eur. Phys. J. A 22 (2004) 37.

[49] M. El Adri, M. Oulne, Eur. Phys. J. Plus 135 (2020) 268.

[50] S. Tagami, J. Matsui, M. Takechi, M. Yahiro, arXiv:2005.13197 [nucl-th].

[51] A.J. Miller, et al., Nat. Phys. 15 (2019) 432.

[52] R.F. Garcia Ruiz, et al., Nat. Phys. 12 (2016) 594.

[53] A. Ozawa, T. Kobayashi, T. Suzuki, K. Yoshida, I. Tanihata, Phys. Rev. Lett. 84 (2000) 5493.

[54] E.K. Warburton, et al., Phys. Rev. C 41 (1990) 1447.

[55] I. Angeli, K. Marinova, J. Phys. G 42 (2015) 055108.

[56] A. Mutschler, et al., Nat. Phys. 13 (2017) 152.

[57] P. Doornenbal, et al., Phys. Lett. B 647 (2007) 237. 
[58] N. Wang, M. Liu, H. Jiang, J.L. Tian, Y.M. Zhao, Phys. Rev. C 91 (2015) 044308.

[59] A.N. Antonov, D.N. Kadrev, M.K. Gaidarov, E. Moya de Guerra, P. Sarriguren, J.M. Udias, V.K. Lukyanov, E.V. Zemlyanaya, G.Z. Krumova, Phys. Rev. C 72 (2005) 044307.

[60] X. Roca-Maza, M. Centelles, F. Salvat, X. Viñas, Phys. Rev. C 87 (2013) 014304.

[61] A. Meucci, M. Vorabbi, C. Giusti, F.D. Pacati, Phys. Rev. C 89 (2014) 034604. 